\title{
Effects of Friction on Heterogeneous Deformation Characteristics of Interstitial-free Steels during the Torsion Stage of High Pressure Torsion
}

\author{
Yuepeng Song ${ }^{1,2}$, Zihan Zhang ${ }^{1}$, Dongsheng Gao ${ }^{1, *}$, Hongmei Zhang ${ }^{1}$ and Hyoung-seop Kim ${ }^{2}$ \\ ${ }^{1}$ Shandong Agricultural University, Mechanical and Electronic Engineering College, Shandong Provincial Key Laboratory of \\ Horticultural Machineries and Equipments, Tai'an 271018, China \\ ${ }^{2}$ Pohang University of Science and Technology, Department of Materials Science and Engineering, Pohang 790784, Korea \\ ${ }^{*}$ Corresponding author
}

\begin{abstract}
The heterogeneous plastic deformation characteristics of HPT processed Interstitial-free steels (IF-steels) and the effects of friction coefficient on heterogeneous plastic deformation characteristics were investigated through the effective strain distribution on the transversal plane of HTP disks. Using DEFORM-3D finite analysis software, the simulation results were obtained firstly, verified by experimental approaches. The deformation characteristic is remarkably heterogeneous in radial and axial direction on the transversal plane of HPT-processed IF steel samples. Along the radial direction, the low value of the effective strain exists in the central position, high value exists in the edge, correspondingly. Along the axial direction, the value of the bottom surface is significantly higher than other surfaces. In addition, friction coefficient plays a significantly role in early torsion stage. More and more heterogeneous plastic deformation characteristics are exhibited following the friction coefficient increase. The experimental results strongly support the simulation conclusions.
\end{abstract}

Keywords-finite element analysis; friction coefficient; heterogeneous deformation; high pressure torsion; if steel

\section{INTRODUCTION}

In recent years, the investigation of ultrafine-grained (UFG) material has been surged tremendously due to UFG material exhibiting superior mechanical properties and machining formability [1-2]. Severe plastic deformation (SPD) has been considered the most successful method for fabricating UFG materials. High Pressure Torsion (HPT) is one of the most effective processes for refining grain in SPD. At present, the researcher had successful prepared $\mathrm{Al}, \mathrm{Mg}, \mathrm{Cu}$, Ti and other material with HPT method [2,4-9]. As the demand for steel in the automobile industry increases gradually, the research on improving IF steels strength by HPT method is gradually increased [6-7].

It has been verified as a currently accepted rule that heterogeneous plastic deformation characteristics occurred in compression stage of HPT process [7,8,10-12]. And parameter like the effects of pressure, turns and friction on torsion stage during the HPT process have been focused. Investigation indicates that the parameters especially the friction coefficient have great influence on the heterogeneous plastic deformation characteristics during the HPT process [6,8-12]. However, it should be noted that, as to the different section of the
HPT-processed IF disks, the effects of friction on heterogeneous plastic deformation characteristics are not reported all over the world.

In this paper, the finite element analysis software DEFORM-3D is used to study the heterogeneous plastic deformation characteristic of the HPT disks at different friction coefficient in the torsion stage. By analyzing the efficient strain distribution of the HPT disks, combined with hardness experience measurement, heterogeneous plastic deformation characteristic in the stage of torsion and effects of friction on heterogeneous deformation are investigated.

\section{ESTABLishment OF FINITE ELEMENT MODEL AND EXPERIMENT DESIGN}

In the HPT process, the disk is limited by the groove of the bottom die, and is compressed by the upper die of pressure, leading to compression deformation in the axial direction. After the end of the process of compression, the disk is loaded torsion by the rotation of the bottom die, and produces an axial compression and shape deformation. The model of the torsion stage is shown in Figure $\mathrm{I}(\mathrm{a})$ and (b).
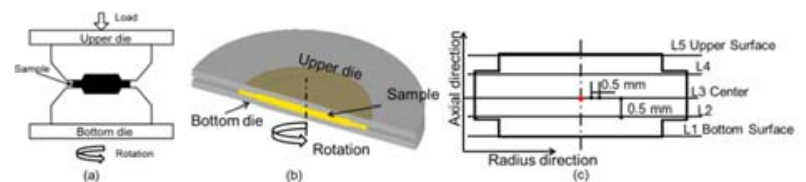

FIGURE I. SCHEMATIC DIAGRAM AND SIMULATION MODEL OF HIGH PRESSURE TORSION PROCESSING IN TORSION STAGE AND MEASUREMENT OF HARDNESS DISTRIBUTION OF DISKS (A) SCHEMATIC DIAGRAM, (B) SIMULATION MODEL (C) TESTING PLANES OF THE HARDNESS

The disks material adopted IF steel (manufactured by the Pohang Steel Company, POSCO, Korea), the main components include $0.008 \% \mathrm{C}, 0.096 \% \mathrm{Mn}, 0.045 \% \mathrm{Al}$, $0.041 \% \mathrm{Ti}, \mathrm{Fe}$ allowance. The HPT-processed IF steel disks were $20 \mathrm{~mm}$ of diameter and $2.0 \mathrm{~mm}$ of thickness, and the initial hardness was about $80 \mathrm{Hv}$. Surface pressure of $2.5 \mathrm{GPa}$ was imposed on the disks at room temperature. The time of compression stage and torsion stage were both conducted at 10 s. For studying the effect of friction coefficient on inhomogeneous deformation of IF steel in torsion stage of 
HPT, the disk surfaces are coated with graphite powder, another did not be disposed as control group.

The graphite powder can form a thin film of graphite on the surface of the disk and reduce the friction coefficient significantly. An FM-700 micro-hardness tester was used to measure the hardness at different positions on the disks. Testing planes of the hardness is show in Figure I(c). Along with the radius direction, from center to edge, adjacent hardness testing pots is at $0.5 \mathrm{~mm}$ intervals.

The finite element software (DEFORM-3D) is applied to simulate the plastic deformation of IF steel disks in HPT process. In the FEM simulation, the initial disks dimension is $20 \mathrm{~mm}$ in diameter and $2 \mathrm{~mm}$ thick. During the simulation process, $0.008 \% \mathrm{C}$ carbon steel material in the DEFORM-3D material library is selected as the physical parameter of the IF steel disks material.

It can regard the model as a rigid model and not need to mesh the model. In order to ensure the accuracy of the simulation results and improve the calculation efficiency, the best number of the initial mesh in the disks was 22000 .

In the compression stage, pressure of $2.5 \mathrm{GPa}$ was developed on the disks. In the torsion stage, the bottom die rotated at $1 \mathrm{rad} / \mathrm{s}$ with a constant pressure of $2.5 \mathrm{GPa}$. The applied friction between die and disks was $0.05,0.1,1,1.5$ and 2 , and the time of compression stage and torsion stage both were $10 \mathrm{~s}$ in all cases. In this paper, the turn adopted the early torsion stage (1/4 turns).

\section{RESULTS AND DisCUSSION}

A. Heterogeneous Distributions of Effective Strain and Hardness

Figure II (a) (f) indicates the Effective Strain distribution of HPT-processed disks at the different stage with finite element simulation approach.

\section{I(a) before high pressure torsion}

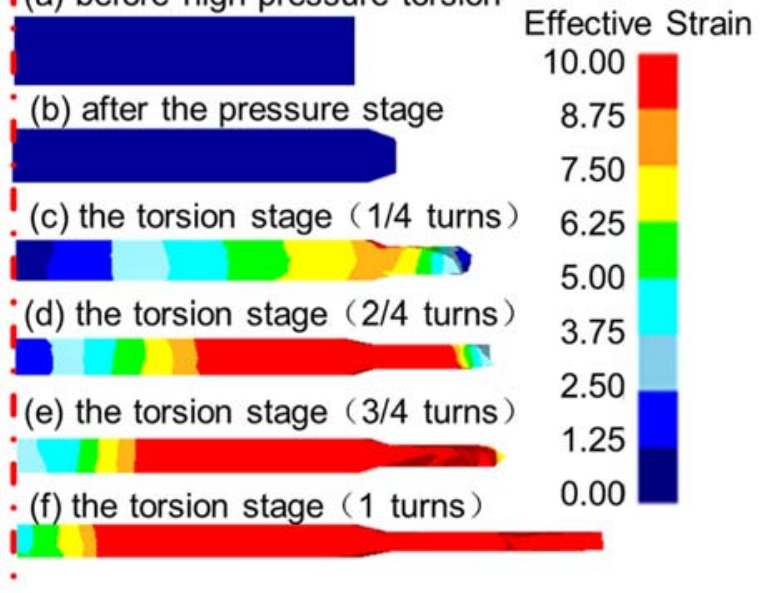

FIGURE II. THICKNESS DISTRIBUTION OF DISKS IN DIFFERENT ANGLES OF TURNS (A) BEFORE HPT (B) AFTER THE PRESSURE STAGE (C) 1/4 TURNS (D) 2/4 TURNS (E)3/4 TURNS (F)1 TURNS
From Figure II(a) to (f), the thickness of disk will be decreased in the whole process of HPT, whether compression or torsion stage. The experimental results clearly show that the disks thickness decreases following the increase of angles of torsion turns. The original thickness of disk is $2 \mathrm{~mm}$, while reduces to $1.9 \mathrm{~mm}$ after pressure stage. As to the early torsion stage(1/4 turns), the decrease rate of disks thickness will be severe changed. With the increase of angles of turns, the thickness of disk continuously reduces. From Figure II, the plastic deformation varied obviously in the early torsion stage( $1 / 4$ turns).

Figure III shows the effective strain distribution on the transversal plane of the HPT-processed IF steel disks in the finite element model (2.5 GPa; $1 / 4$ turns, $\mu=1.5)$. In Figure III(a), the effective strain of disk in different position of center, middle, and edge were about $1,4,8$, correspondingly. The value of effective strain of disks is significantly increased along the radial direction. Within a distance of $5 \mathrm{~mm}$ from center of disks, the values on all testing plane are about same. In Figure III(b), the enlarged partial detail figure $(5 \mathrm{~mm} \sim 10$ $\mathrm{mm}$ ) exhibited that the value of effective strain on the bottom surface is above others. And this regular is more and more obviously exhibited from center to edge. Thus, there is deformation lagging character in early HPT stage.

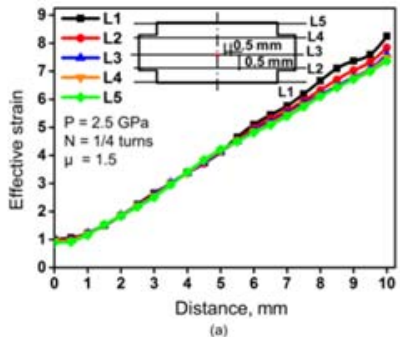

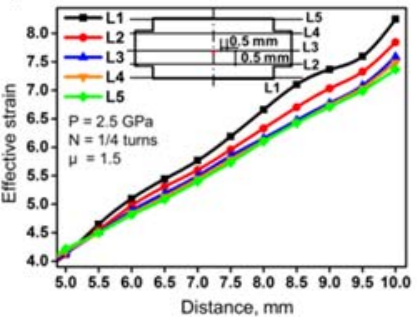

(b)
FIGURE III. EFFECTIVE STRAIN DISTRIBUTION OF DISKS IN FINITE ELEMENT MODEL

\section{B. Heterogeneous Distributions of Hardness}

Figure IV shows the hardness distribution on the transversal plane of the early torsion stage disks.

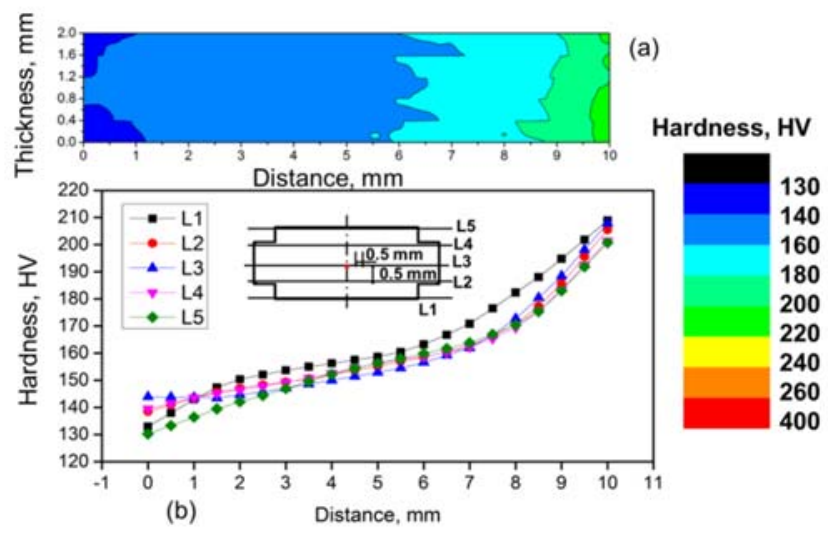

FIGURE IV. HARDNESS DISTRIBUTION OF DISKS (A) EFFECTIVE STRAIN IN FINITE ELEMENT MODEL (B) HARDNESS IN EXPERIENCE MODEL 
As the Figure IV(a) shown, the value of hardness is heterogeneous in the early torsion stage of the HPT process. From Figure IV(a), the hardness values in the center, middle, and edge of the disks were about $130 \mathrm{Hv}, 180 \mathrm{Hv}$ and $220 \mathrm{Hv}$. The figure indicates significantly that low hardness values in the center and the high at the edge along radius direction. Besides, hardness distribution is heterogeneous in axial direction. Compared with the bottom surface, the hardness values is lower in other test plane after torsion, especially test plane which is close to the upper surface.

In Figure IV(b), the hardness gradually increases from center to edge. Within a distance of $2 \mathrm{~mm}$ from the center, the hardness is above $130 \mathrm{HV}$. Compared with the initial state, the hardness value is increasing about 60 percent after the process of HPT. During $2 \mathrm{~mm} \sim 6 \mathrm{~mm}$ from the center, the hardness values sustained growth, and the bottom is above others. That is to say, large deformation proceeds gradually from the edge to the center along the radial direction and from the bottom surface to the upper surfaces along the axial direction.

This also indicates the conclusion that deformation lagging characteristics of IF steel disks are in the early torsion stage of HPT [12]. The hardness values are agreed with effective strain value Hence, the reliability of this computer simulation is verified.

\section{Effect of Friction on the Plastic Deformation in the Torsion Stage}

Figure V obviously shows the effective strain distribution on half cross-sectional planes at five friction coefficients of $0.05,0.1,1,1.5$ and 2 under $2.5 \mathrm{GPa}$ and $1 / 4$ turns.

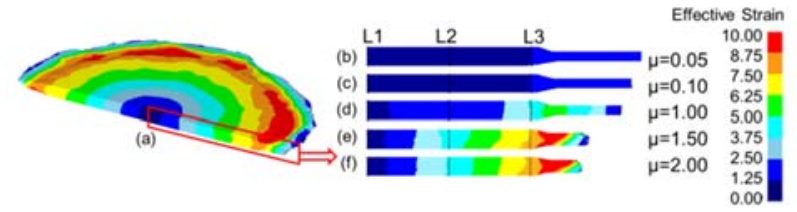

FIGURE V. THE EFFECTIVE STRAIN DISTRIBUTION OF DISKS IN FINITE ELEMENT MODEL WITH DIFFERENT FRICTION (A) EFFECTIVE STRAIN DISTRIBUTION (B) 0.05, (C) 0.1, (D) 1, AND (E) $1.5,(\mathrm{~F}) 2$

The distribution of effective strain in the disks is more heterogeneous as the friction coefficient increases showed in figure. From Figure V(b), (c), at the low friction coefficient, the value of effective strain is less heterogeneous along radial direction in the early torsion stage of the HPT process and the maximum value is 2.5. At the friction coefficient 1 , the distribution of effective strain is more heterogeneous than at low friction coefficient. In Figure V(d), the effective strain is 2.5 at middle of disks and 5 at edge. Compares with low friction coefficient, the value is increased about 50 percent. As the friction coefficient increase, the heterogeneous distribution is more visible. As Figure V(e), the effective strain on half cross-sectional planes increases with the distance from bottom surface of disks.

Compared with center and middle of disks, the deformation lagging character is more obvious. This regular also exhibits the situation that friction is 2 . The results indicate that the value of effective strain is higher as friction coefficient increasing, and the distributions of effective strain are heterogeneous. And the deformation lagging characteristic is more significantly especially in edge and bottom surface. The conclusion is also exhibited in the Figure III.

\section{Effect of Friction on the Plastic Deformation in the Torsion Stage}

Figure VI shows the HPT disk and its hardness distribution on the axisymmetric section testing plane with different friction. Figure VI(a) and (b) shows the disk with low and high friction. Figure VI(c) shows the disk in experience.

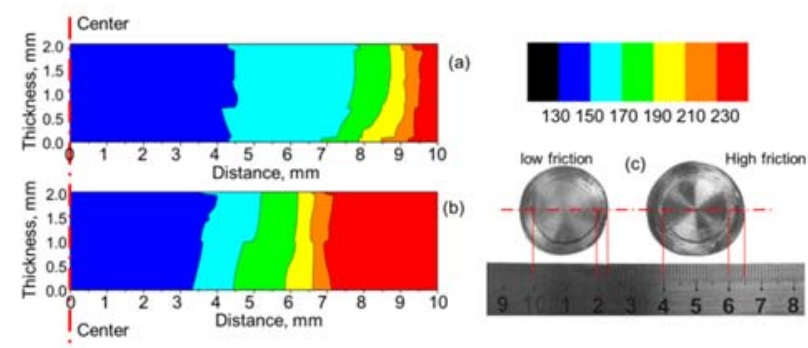

FIGURE VI. THE THICKNESS DISTRIBUTION OF DISKS WITH DIFFERENT FRICTION (A) LOW FRICTION (B) HIGH FRICTION (C) DISK IN EXPERIENCE

From Figure VI(c), we can see the disk after HPT process with different friction coefficient. The figure clearly indicated that the heterogeneous distribution is more and more visible with the increase of fiction coefficient. As Figure VI (a), the value hardness, $150 \mathrm{Hv}$, appear at $4.5 \mathrm{~mm}$ with low friction coefficient and at $3.5 \mathrm{~mm}$ with high friction coefficient. This regular also appears at $230 \mathrm{Hv}$. Thus, the heterogeneous distribution is influenced by friction coefficient.

The results indicate that the conclusion in finite element analysis is same as the test. The simulations results can be strongly supported by experimental ones, which means that simulation have a convincing reliability.

\section{SUMMARY}

In the study, the characteristic of heterogeneous plastic deformation of HPT-processed IF steel disks and the effect of friction coefficient on heterogeneous plastic deformation were investigated using experimental and simulation approaches.

The simulation and experimental results indicated that the value of effective strain and hardness was heterogeneous in the early torsion stage of the HPT process. Along the radial direction, the low value exists in the center zone, while high value in the edge. Along the axial direction, the values of the bottom surface were significantly higher than other surfaces.

More and more heterogeneous plastic deformation characteristics were exhibited following the friction coefficient increase. The experimental results strongly supported the simulation conclusions.

\section{ACKNOWLEDGEMENT}

This work was supported by Funds of national key research and development for 13th year plan (2018YFD0700604, 2016YFD0701701), Innovation team 
fund for fruit industry of modern agricultural technology system in Shandong Province (SDAIT-06-12, SDAIT-06-1), Research project-2017 on intelligent agricultural mechanization equipment of Shandong Province(2017YF003) and Funds of Shandong "Double Tops" programs (SYL2017XTTD07).

\section{REFERENCES}

[1] Faraji G, Kim H S. Review of principles and methods of severe plastic deformation for producing ultrafine-grained tubes, J. Metal Science Journal, 33(2016) 905-923.

[2] Park B H, Um H Y, Kim J G, et al. Large deformation behavior of twin-induced plasticity steels under high-pressure torsion, J. Metals \& Materials International, 22 (2016) 1003-1008.

[3] Um H Y, Park B H, Ahn D H, et al. Mechanical and biological behavior of ultrafine-grained $\mathrm{Ti}$ alloy aneurysm clip processed using high-pressure torsion, J. Journal of the Mechanical Behavior of Biomedical Materials, 68(2017) 203-209.

[4] Ito Y, Edalati K, Horita Z. High-pressure torsion of aluminum with ultrahigh purity $(99.9999 \%)$ and occurrence of inverse Hall-Petch relationship, J. Materials Science \& Engineering A, 679 (2017) 428-434.

[5] Jie Xu, Jianwei Li, Chuanting Wang, et al. Evidence for an early softening behavior in pure copper processed by high-pressure torsion, J. Journal of Materials Science, 51 (2016) 1923-1930.

[6] Saray O, Purcek G, Karaman I, et al. Formability of Ultrafine-Grained Interstitial-Free Steels, J. Metallurgical \& Materials Transactions A, 44(2013) 4194-4206

[7] Yuepeng Song, Wenke Wang, Dongsheng Gao, et al. Hardness and microstructure of interstitial free steels in the early stage of high-pressure torsion, J. Journal of Materials Science,48(2013) 4698-4704.

[8] Yuepeng Song, Wenke Wang, Dong Jun Lee, et al. Thickness inhomogeneity in hardness and microstructure of copper after the compressive stage in high-pressure torsion, J. Metals \& Materials International, 21(2015):7-13.

[9] Jahedi M, Beyerlein I J, Paydar M H, et al. Effects of pressure and number of turns on microstructural homogeneity developed in High-Pressure double Torsion, J. Metallurgical \& Materials Transactions A, 48(2017) 1249-1263.

[10] Yuepeng Song, Miaomiao Chen, Baoyan Xu, et al. Effects of Friction and Anvil Design on Plastic Deformation during the Compression Stage of High-Pressure Torsion, J. Korean Journal of Metals and Materials, 4 (2016) 831-837.

[11] Yuepeng Song, Miaomiao Chen, Baoyan Xu, et al. Effect of Revolution on Inhomogeneous Deformation of IF Steel in High Pressure Torsion, J. Materials Sciences and Applications, 7 (2016) 673-679.

[12] Yuepeng Song, Zihan Zhang, Dongsheng Gao, et al. Deformation lagging characteristics of IF steel disks in the plastic deformation process of high pressure torsion, J. Sci Sin Tech, 48(2017) 154-160. 